\title{
Linear Map of Matrices
}

\author{
Karol Pąk \\ Institute of Computer Science \\ University of Białystok \\ Poland
}

Summary. The paper is concerned with a generalization of concepts introduced in [13], i.e. introduced are matrices of linear transformations over a finitedimensional vector space. Introduced are linear transformations over a finitedimensional vector space depending on a given matrix of the transformation. Finally, I prove that the rank of linear transformations over a finite-dimensional vector space is the same as the rank of the matrix of that transformation.

MML identifier: MATRLIN2, version: 7.9.03 4.104.1021

The notation and terminology used here are introduced in the following papers: [24], [2], [3], [9], [25], [6], [8], [7], [4], [23], [19], [12], [10], [27], [28], [26], [22], [20], [18], [29], [5], [15], [13], [17], [11], [14], [21], [1], and [16].

\section{Preliminaries}

We adopt the following rules: $i, j, m, n$ are natural numbers, $K$ is a field, and $a$ is an element of $K$.

One can prove the following propositions:

(1) Let $V$ be a vector space over $K, W_{1}, W_{2}, W_{12}$ be subspaces of $V$, and $U_{1}$, $U_{2}$ be subspaces of $W_{12}$. If $U_{1}=W_{1}$ and $U_{2}=W_{2}$, then $W_{1} \cap W_{2}=U_{1} \cap U_{2}$ and $W_{1}+W_{2}=U_{1}+U_{2}$.

(2) Let $V$ be a vector space over $K$ and $W_{1}, W_{2}$ be subspaces of $V$. Suppose $W_{1} \cap W_{2}=\mathbf{0}_{V}$. Let $B_{1}$ be a linearly independent subset of $W_{1}$ and $B_{2}$ be a linearly independent subset of $W_{2}$. Then $B_{1} \cup B_{2}$ is a linearly independent subset of $W_{1}+W_{2}$. 
(3) Let $V$ be a vector space over $K$ and $W_{1}, W_{2}$ be subspaces of $V$. Suppose $W_{1} \cap W_{2}=\mathbf{0}_{V}$. Let $B_{1}$ be a basis of $W_{1}$ and $B_{2}$ be a basis of $W_{2}$. Then $B_{1} \cup B_{2}$ is a basis of $W_{1}+W_{2}$.

(4) For every finite dimensional vector space $V$ over $K$ holds every ordered basis of $\Omega_{V}$ is an ordered basis of $V$.

(5) Let $V_{1}$ be a vector space over $K$ and $A$ be a finite subset of $V_{1}$. If $\operatorname{dim}(\operatorname{Lin}(A))=\operatorname{card} A$, then $A$ is linearly independent.

(6) For every vector space $V$ over $K$ and for every finite subset $A$ of $V$ holds $\operatorname{dim}(\operatorname{Lin}(A)) \leq \operatorname{card} A$.

\section{More on the Product of Finite Sequence of Scalars and VECTORS}

For simplicity, we follow the rules: $V_{1}, V_{2}, V_{3}$ are finite dimensional vector spaces over $K, f$ is a function from $V_{1}$ into $V_{2}, b_{1}, b_{1}^{\prime}$ are ordered bases of $V_{1}$, $B_{1}$ is a finite sequence of elements of $V_{1}, b_{2}$ is an ordered basis of $V_{2}, B_{2}$ is a finite sequence of elements of $V_{2}, B_{3}$ is a finite sequence of elements of $V_{3}, v_{1}$, $w_{1}$ are elements of $V_{1}, R, R_{1}, R_{2}$ are finite sequences of elements of $V_{1}$, and $p$, $p_{1}, p_{2}$ are finite sequences of elements of $K$.

We now state a number of propositions:

(7) $\operatorname{lmlt}\left(p_{1}+p_{2}, R\right)=\operatorname{lmlt}\left(p_{1}, R\right)+\operatorname{lmlt}\left(p_{2}, R\right)$.

(8) $\operatorname{lmlt}\left(p, R_{1}+R_{2}\right)=\operatorname{lmlt}\left(p, R_{1}\right)+\operatorname{lmlt}\left(p, R_{2}\right)$.

(9) If len $p_{1}=$ len $R_{1}$ and len $p_{2}=\operatorname{len} R_{2}$, then $\operatorname{lmlt}\left(p_{1} \frown p_{2}, R_{1} \frown R_{2}\right)=$ $\left(\operatorname{lmlt}\left(p_{1}, R_{1}\right)\right)^{\frown} \operatorname{lmlt}\left(p_{2}, R_{2}\right)$.

(10) If len $R_{1}=$ len $R_{2}$, then $\sum\left(R_{1}+R_{2}\right)=\left(\sum R_{1}\right)+\sum R_{2}$.

(11) $\sum \operatorname{lmlt}(\operatorname{len} R \mapsto a, R)=a \cdot \sum R$.

(12) $\sum \operatorname{lmlt}\left(p, \operatorname{len} p \mapsto v_{1}\right)=\left(\sum p\right) \cdot v_{1}$.

(13) $\sum \operatorname{lmlt}(a \cdot p, R)=a \cdot \sum \operatorname{lmlt}(p, R)$.

(14) Let $B_{1}$ be a finite sequence of elements of $V_{1}, W_{1}$ be a subspace of $V_{1}$, and $B_{2}$ be a finite sequence of elements of $W_{1}$. If $B_{1}=B_{2}$, then $\operatorname{lmlt}\left(p, B_{1}\right)=\operatorname{lmlt}\left(p, B_{2}\right)$.

(15) Let $B_{1}$ be a finite sequence of elements of $V_{1}, W_{1}$ be a subspace of $V_{1}$, and $B_{2}$ be a finite sequence of elements of $W_{1}$. If $B_{1}=B_{2}$, then $\sum B_{1}=\sum B_{2}$.

(16) If $i \in \operatorname{dom} R$, then $\sum \operatorname{lmlt}\left(\operatorname{Line}\left(I_{K}^{\operatorname{len} R \times \operatorname{len} R}, i\right), R\right)=R_{i}$.

\section{More on the Decomposition of a Vector in a Basis}

We now state a number of propositions:

$$
v_{1}+w_{1} \rightarrow b_{1}=\left(v_{1} \rightarrow b_{1}\right)+\left(w_{1} \rightarrow b_{1}\right) .
$$


(18) $a \cdot v_{1} \rightarrow b_{1}=a \cdot\left(v_{1} \rightarrow b_{1}\right)$.

(19) If $i \in \operatorname{dom} b_{1}$, then $\left(b_{1}\right)_{i} \rightarrow b_{1}=\operatorname{Line}\left(I_{K}^{\operatorname{len} b_{1} \times \operatorname{len} b_{1}}, i\right)$.

(20) $\quad 0_{\left(V_{1}\right)} \rightarrow b_{1}=$ len $b_{1} \mapsto 0_{K}$.

(21) $\operatorname{len} b_{1}=\operatorname{dim}\left(V_{1}\right)$.

(22)(i) $\operatorname{rng}\left(b_{1}\lceil m)\right.$ is a linearly independent subset of $V_{1}$, and

(ii) for every subset $A$ of $V_{1}$ such that $A=\operatorname{rng}\left(b_{1}\lceil m)\right.$ holds $b_{1}\lceil m$ is an ordered basis of $\operatorname{Lin}(A)$.

(23)(i) $\operatorname{rng}\left(\left(b_{1}\right)_{\lfloor m}\right)$ is a linearly independent subset of $V_{1}$, and

(ii) for every subset $A$ of $V_{1}$ such that $A=\operatorname{rng}\left(\left(b_{1}\right)_{\lfloor m}\right)$ holds $\left(b_{1}\right)_{\lfloor m}$ is an ordered basis of $\operatorname{Lin}(A)$.

(24) Let $W_{1}, W_{2}$ be subspaces of $V_{1}$. Suppose $W_{1} \cap W_{2}=\mathbf{0}_{\left(V_{1}\right)}$. Let $b_{1}$ be an ordered basis of $W_{1}, b_{2}$ be an ordered basis of $W_{2}$, and $b$ be an ordered basis of $W_{1}+W_{2}$. Suppose $b=b_{1} \frown b_{2}$. Let $v, v_{1}, v_{2}$ be vectors of $W_{1}+W_{2}$, $w_{1}$ be a vector of $W_{1}$, and $w_{2}$ be a vector of $W_{2}$. If $v=v_{1}+v_{2}$ and $v_{1}=w_{1}$ and $v_{2}=w_{2}$, then $v \rightarrow b=\left(w_{1} \rightarrow b_{1}\right)^{\frown}\left(w_{2} \rightarrow b_{2}\right)$.

(25) Let $W_{1}$ be a subspace of $V_{1}$. Suppose $W_{1}=\Omega_{\left(V_{1}\right)}$. Let $w$ be a vector of $W_{1}, v$ be a vector of $V_{1}$, and $w_{1}$ be an ordered basis of $W_{1}$. If $v=w$ and $b_{1}=w_{1}$, then $v \rightarrow b_{1}=w \rightarrow w_{1}$.

(26) Let $W_{1}, W_{2}$ be subspaces of $V_{1}$. Suppose $W_{1} \cap W_{2}=\mathbf{0}_{\left(V_{1}\right)}$. Let $w_{1}$ be an ordered basis of $W_{1}$ and $w_{2}$ be an ordered basis of $W_{2}$. Then $w_{1} \frown w_{2}$ is an ordered basis of $W_{1}+W_{2}$.

\section{Properties of Matrices of Linear Transformations}

Let us consider $K, V_{1}, V_{2}, f, B_{1}, b_{2}$. Then $\operatorname{AutMt}\left(f, B_{1}, b_{2}\right)$ is a matrix over $K$ of dimension len $B_{1} \times \operatorname{len} b_{2}$.

Let $S$ be a 1-sorted structure and let $R$ be a binary relation. The functor $R \uparrow S$ is defined as follows:

(Def. 1) $\quad R \uparrow S=R \uparrow$ the carrier of $S$.

The following proposition is true

(27) Let $f$ be a linear transformation from $V_{1}$ to $V_{2}, W_{1}, W_{2}$ be subspaces of $V_{1}$, and $U_{1}, U_{2}$ be subspaces of $V_{2}$. Suppose if $\operatorname{dim}\left(W_{1}\right)=0$, then $\operatorname{dim}\left(U_{1}\right)=0$ and if $\operatorname{dim}\left(W_{2}\right)=0$, then $\operatorname{dim}\left(U_{2}\right)=0$ and $V_{2}$ is the direct sum of $U_{1}$ and $U_{2}$. Let $f_{1}$ be a linear transformation from $W_{1}$ to $U_{1}$ and $f_{2}$ be a linear transformation from $W_{2}$ to $U_{2}$. Suppose $f_{1}=f \uparrow W_{1}$ and $f_{2}=f\left\lceil W_{2}\right.$. Let $w_{1}$ be an ordered basis of $W_{1}, w_{2}$ be an ordered basis of $W_{2}, u_{1}$ be an ordered basis of $U_{1}$, and $u_{2}$ be an ordered basis of $U_{2}$. Suppose $w_{1} \frown w_{2}=b_{1}$ and $u_{1} \frown u_{2}=b_{2}$. Then $\operatorname{AutMt}\left(f, b_{1}, b_{2}\right)=$ the $0_{K}$-block diagonal of $\left\langle\operatorname{AutMt}\left(f_{1}, w_{1}, u_{1}\right), \operatorname{AutMt}\left(f_{2}, w_{2}, u_{2}\right)\right\rangle$. 
Let us consider $K, V_{1}, V_{2}$, let $f$ be a function from $V_{1}$ into $V_{2}$, let $B_{1}$ be a finite sequence of elements of $V_{1}$, and let $b_{2}$ be an ordered basis of $V_{2}$. Let us assume that len $B_{1}=\operatorname{len} b_{2}$. The functor $\operatorname{AutEqMt}\left(f, B_{1}, b_{2}\right)$ yielding a matrix over $K$ of dimension len $B_{1} \times$ len $B_{1}$ is defined by:

(Def. 2) $\operatorname{AutEqMt}\left(f, B_{1}, b_{2}\right)=\operatorname{AutMt}\left(f, B_{1}, b_{2}\right)$.

The following propositions are true:

(28) AutMt $\left(\mathrm{id}_{\left(V_{1}\right)}, b_{1}, b_{1}\right)=I_{K}^{\operatorname{len} b_{1} \times \operatorname{len} b_{1}}$.

(29) AutEqMt( $\left(\mathrm{id}_{\left(V_{1}\right)}, b_{1}, b_{1}^{\prime}\right)$ is invertible and $\operatorname{AutEqMt}\left(\operatorname{id}_{\left(V_{1}\right)}, b_{1}^{\prime}, b_{1}\right)=$ $\left(\operatorname{AutEqMt}\left(\operatorname{id}_{\left(V_{1}\right)}, b_{1}, b_{1}^{\prime}\right)\right)^{\smile}$.

(30) If len $p_{1}=\operatorname{len} p_{2}$ and $\operatorname{len} p_{1}=\operatorname{len} B_{1}$ and len $p_{1}>0$ and $j \in \operatorname{dom} b_{1}$ and for every $i$ such that $i \in \operatorname{dom} p_{2}$ holds $p_{2}(i)=\left(\left(B_{1}\right)_{i} \rightarrow b_{1}\right)(j)$, then $p_{1} \cdot p_{2}=\left(\sum \operatorname{lmlt}\left(p_{1}, B_{1}\right) \rightarrow b_{1}\right)(j)$.

(31) If len $b_{1}>0$ and $f$ is linear, then $\operatorname{LineVec} 2 \mathrm{Mx}\left(v_{1} \rightarrow b_{1}\right)$. $\operatorname{AutMt}\left(f, b_{1}, b_{2}\right)=\operatorname{LineVec} 2 \mathrm{Mx}\left(f\left(v_{1}\right) \rightarrow b_{2}\right)$.

\section{Linear Transformations of Matrices}

Let us consider $K, V_{1}, V_{2}, b_{1}, B_{2}$ and let $M$ be a matrix over $K$ of dimension len $b_{1} \times$ len $B_{2}$. The functor $\operatorname{Mx} 2 \operatorname{Tran}\left(M, b_{1}, B_{2}\right)$ yielding a function from $V_{1}$ into $V_{2}$ is defined by:

(Def. 3) For every vector $v$ of $V_{1}$ holds $\left(\operatorname{Mx} 2 \operatorname{Tran}\left(M, b_{1}, B_{2}\right)\right)(v)=$ $\sum \operatorname{lmlt}\left(\operatorname{Line}\left(\operatorname{LineVec} 2 \mathrm{Mx}\left(v \rightarrow b_{1}\right) \cdot M, 1\right), B_{2}\right)$.

Next we state two propositions:

(32) For every matrix $M$ over $K$ of dimension len $b_{1} \times \operatorname{len} b_{2}$ such that len $b_{1}>0$ holds LineVec2Mx $\left(\left(\operatorname{Mx} 2 \operatorname{Tran}\left(M, b_{1}, b_{2}\right)\right)\left(v_{1}\right) \rightarrow b_{2}\right)=$ LineVec $2 \mathrm{Mx}\left(v_{1} \rightarrow b_{1}\right) \cdot M$.

(33) For every matrix $M$ over $K$ of dimension len $b_{1} \times$ len $B_{2}$ such that len $b_{1}=0$ holds $\left(\operatorname{Mx} 2 \operatorname{Tran}\left(M, b_{1}, B_{2}\right)\right)\left(v_{1}\right)=0_{\left(V_{2}\right)}$.

Let us consider $K, V_{1}, V_{2}, b_{1}, B_{2}$ and let $M$ be a matrix over $K$ of dimension len $b_{1} \times$ len $B_{2}$. Then $\operatorname{Mx} 2 \operatorname{Tran}\left(M, b_{1}, B_{2}\right)$ is a linear transformation from $V_{1}$ to $V_{2}$.

Next we state three propositions:

(34) If $f$ is linear, then $\operatorname{Mx} 2 \operatorname{Tran}\left(\operatorname{AutMt}\left(f, b_{1}, b_{2}\right), b_{1}, b_{2}\right)=f$.

(35) For all matrices $A, B$ over $K$ such that $i \in \operatorname{dom} A$ and width $A=\operatorname{len} B$ holds LineVec2Mx Line $(A, i) \cdot B=\operatorname{LineVec} 2 \mathrm{Mx} \operatorname{Line}(A \cdot B, i)$.

(36) For every matrix $M$ over $K$ of dimension len $b_{1} \times$ len $b_{2}$ holds $\operatorname{AutMt}\left(\mathrm{Mx} 2 \operatorname{Tran}\left(M, b_{1}, b_{2}\right), b_{1}, b_{2}\right)=M$.

Let us consider $n, m, K$, let $A$ be a matrix over $K$ of dimension $n \times m$, and let $B$ be a matrix over $K$. Then $A+B$ is a matrix over $K$ of dimension $n \times m$. 
The following propositions are true:

(37) For all matrices $A, B$ over $K$ of dimension len $b_{1} \times$ len $B_{2}$ holds $\operatorname{Mx} 2 \operatorname{Tran}\left(A+B, b_{1}, B_{2}\right)=\operatorname{Mx} 2 \operatorname{Tran}\left(A, b_{1}, B_{2}\right)+\operatorname{Mx} 2 \operatorname{Tran}\left(B, b_{1}, B_{2}\right)$.

(38) For every matrix $A$ over $K$ of dimension len $b_{1} \times \operatorname{len} B_{2}$ holds $a$. $\operatorname{Mx} 2 \operatorname{Tran}\left(A, b_{1}, B_{2}\right)=\operatorname{Mx} 2 \operatorname{Tran}\left(a \cdot A, b_{1}, B_{2}\right)$.

(39) For all matrices $A, B$ over $K$ of dimension len $b_{1} \times$ len $b_{2}$ such that $\operatorname{Mx} 2 \operatorname{Tran}\left(A, b_{1}, b_{2}\right)=\operatorname{Mx} 2 \operatorname{Tran}\left(B, b_{1}, b_{2}\right)$ holds $A=B$.

(40) Let $A$ be a matrix over $K$ of dimension len $b_{1} \times \operatorname{len} b_{2}$ and $B$ be a matrix over $K$ of dimension len $b_{2} \times$ len $B_{3}$. Suppose width $A=$ len $B$. Let $A_{1}$ be a matrix over $K$ of dimension len $b_{1} \times \operatorname{len} B_{3}$. If $A_{1}=A \cdot B$, then $\operatorname{Mx} 2 \operatorname{Tran}\left(A_{1}, b_{1}, B_{3}\right)=\operatorname{Mx} 2 \operatorname{Tran}\left(B, b_{2}, B_{3}\right) \cdot \operatorname{Mx} 2 \operatorname{Tran}\left(A, b_{1}, b_{2}\right)$.

(41) Let $A$ be a matrix over $K$ of dimension len $b_{1} \times \operatorname{len} b_{2}$. Suppose len $b_{1}>0$ and len $b_{2}>0$. Then $v_{1} \in \operatorname{ker} \operatorname{Mx} 2 \operatorname{Tran}\left(A, b_{1}, b_{2}\right)$ if and only if $v_{1} \rightarrow b_{1} \in$ the space of solutions of $A^{\mathrm{T}}$.

(42) $\quad V_{1}$ is trivial iff $\operatorname{dim}\left(V_{1}\right)=0$.

(43) Let $V_{1}, V_{2}$ be vector spaces over $K$ and $f$ be a linear transformation from $V_{1}$ to $V_{2}$. Then $f$ is one-to-one if and only if $\operatorname{ker} f=\mathbf{0}_{\left(V_{1}\right)}$.

Let us consider $K$ and let $V_{1}$ be a vector space over $K$. Then $\operatorname{id}_{\left(V_{1}\right)}$ is a linear transformation from $V_{1}$ to $V_{1}$.

Let us consider $K$, let $V_{1}, V_{2}$ be vector spaces over $K$, and let $f, g$ be linear transformations from $V_{1}$ to $V_{2}$. Then $f+g$ is a linear transformation from $V_{1}$ to $V_{2}$.

Let us consider $K$, let $V_{1}, V_{2}$ be vector spaces over $K$, let $f$ be a linear transformation from $V_{1}$ to $V_{2}$, and let us consider $a$. Then $a \cdot f$ is a linear transformation from $V_{1}$ to $V_{2}$.

Let us consider $K$, let $V_{1}, V_{2}, V_{3}$ be vector spaces over $K$, let $f_{3}$ be a linear transformation from $V_{1}$ to $V_{2}$, and let $f_{4}$ be a linear transformation from $V_{2}$ to $V_{3}$. Then $f_{4} \cdot f_{3}$ is a linear transformation from $V_{1}$ to $V_{3}$.

One can prove the following propositions:

(44) For every matrix $A$ over $K$ of dimension len $b_{1} \times$ len $b_{2}$ such that $\operatorname{rk}(A)=$ len $b_{1}$ holds $\operatorname{Mx} 2 \operatorname{Tran}\left(A, b_{1}, b_{2}\right)$ is one-to-one.

(45) $\operatorname{MX2FinS}\left(I_{K}^{n \times n}\right)$ is an ordered basis of the $n$-dimension vector space over $K$.

(46) Let $M$ be an ordered basis of the len $b_{2}$-dimension vector space over $K$. Suppose $M=\operatorname{MX} 2 \mathrm{FinS}\left(I_{K}^{\text {len } b_{2} \times \operatorname{len} b_{2}}\right)$. Let $v_{1}$ be a vector of the len $b_{2^{-}}$ dimension vector space over $K$. Then $v_{1} \rightarrow M=v_{1}$.

(47) Let $M$ be an ordered basis of the len $b_{2}$-dimension vector space over $K$. Suppose $M=\operatorname{MX} 2 \mathrm{FinS}\left(I_{K}^{\text {len } b_{2} \times \text { len } b_{2}}\right)$. Let $A$ be a matrix over $K$ of dimension len $b_{1} \times \operatorname{len} M$. If $A=\operatorname{AutMt}\left(f, b_{1}, b_{2}\right)$ and $f$ is linear, then $\left(\operatorname{Mx} 2 \operatorname{Tran}\left(A, b_{1}, M\right)\right)\left(v_{1}\right)=f\left(v_{1}\right) \rightarrow b_{2}$. 
Let $K$ be an add-associative right zeroed right complementable Abelian associative well unital distributive non empty double loop structure, let $V_{1}, V_{2}$ be Abelian add-associative right zeroed right complementable vector space-like non empty vector space structures over $K$, let $W$ be a subspace of $V_{1}$, and let $f$ be a function from $V_{1}$ into $V_{2}$. Then $f \uparrow W$ is a function from $W$ into $V_{2}$.

Let $K$ be a field, let $V_{1}, V_{2}$ be vector spaces over $K$, let $W$ be a subspace of $V_{1}$, and let $f$ be a linear transformation from $V_{1}$ to $V_{2}$. Then $f \nmid W$ is a linear transformation from $W$ to $V_{2}$.

\section{The Main Theorems}

The following propositions are true:

(48) For every linear transformation $f$ from $V_{1}$ to $V_{2}$ holds $\operatorname{rank} f=$ $\operatorname{rk}\left(\operatorname{AutMt}\left(f, b_{1}, b_{2}\right)\right)$.

(49) For every matrix $M$ over $K$ of dimension len $b_{1} \times$ len $b_{2}$ holds $\operatorname{rank} \operatorname{Mx} 2 \operatorname{Tran}\left(M, b_{1}, b_{2}\right)=\operatorname{rk}(M)$.

(50) For every linear transformation $f$ from $V_{1}$ to $V_{2}$ such that $\operatorname{dim}\left(V_{1}\right)=$ $\operatorname{dim}\left(V_{2}\right)$ holds ker $f$ is non trivial iff Det $\operatorname{AutEqMt}\left(f, b_{1}, b_{2}\right)=0_{K}$.

(51) Let $f$ be a linear transformation from $V_{1}$ to $V_{2}$ and $g$ be a linear transformation from $V_{2}$ to $V_{3}$. If $g\lceil\operatorname{im} f$ is one-to-one, then $\operatorname{rank}(g \cdot f)=\operatorname{rank} f$ and $\operatorname{nullity}(g \cdot f)=$ nullity $f$.

\section{REFERENCES}

[1] Jesse Alama. The rank+nullity theorem. Formalized Mathematics, 15(3):137-142, 2007.

[2] Grzegorz Bancerek. Cardinal numbers. Formalized Mathematics, 1(2):377-382, 1990.

[3] Grzegorz Bancerek. The fundamental properties of natural numbers. Formalized Mathematics, 1(1):41-46, 1990.

[4] Grzegorz Bancerek and Krzysztof Hryniewiecki. Segments of natural numbers and finite sequences. Formalized Mathematics, 1(1):107-114, 1990.

[5] Czesław Byliński. Binary operations applied to finite sequences. Formalized Mathematics, 1(4):643-649, 1990.

[6] Czesław Byliński. Functions and their basic properties. Formalized Mathematics, 1(1):5565,1990 .

[7] Czesław Byliński. Functions from a set to a set. Formalized Mathematics, 1(1):153-164, 1990.

[8] Czesław Byliński. Partial functions. Formalized Mathematics, 1(2):357-367, 1990.

[9] Agata Darmochwał. Finite sets. Formalized Mathematics, 1(1):165-167, 1990.

[10] Katarzyna Jankowska. Matrices. Abelian group of matrices. Formalized Mathematics, 2(4):475-480, 1991.

[11] Jarosław Kotowicz. Functions and finite sequences of real numbers. Formalized Mathematics, 3(2):275-278, 1992.

[12] Eugeniusz Kusak, Wojciech Leończuk, and Michał Muzalewski. Abelian groups, fields and vector spaces. Formalized Mathematics, 1(2):335-342, 1990.

[13] Robert Milewski. Associated matrix of linear map. Formalized Mathematics, 5(3):339$345,1996$.

[14] Michał Muzalewski. Rings and modules - part II. Formalized Mathematics, 2(4):579-585, 1991. 
[15] Karol Pạk. Basic properties of the rank of matrices over a field. Formalized Mathematics, 15(4):199-211, 2007.

[16] Karol Pąk. Block diagonal matrices. Formalized Mathematics, 16(3):259-267, 2008.

[17] Karol Pąk. Solutions of linear equations. Formalized Mathematics, 16(1):81-90, 2008.

[18] Wojciech A. Trybulec. Basis of vector space. Formalized Mathematics, 1(5):883-885, 1990.

[19] Wojciech A. Trybulec. Groups. Formalized Mathematics, 1(5):821-827, 1990.

[20] Wojciech A. Trybulec. Linear combinations in vector space. Formalized Mathematics, 1(5):877-882, 1990.

[21] Wojciech A. Trybulec. Operations on subspaces in vector space. Formalized Mathematics, 1(5):871-876, 1990.

[22] Wojciech A. Trybulec. Subspaces and cosets of subspaces in vector space. Formalized Mathematics, 1(5):865-870, 1990.

[23] Wojciech A. Trybulec. Vectors in real linear space. Formalized Mathematics, 1(2):291-296, 1990.

[24] Zinaida Trybulec. Properties of subsets. Formalized Mathematics, 1(1):67-71, 1990.

[25] Edmund Woronowicz. Relations and their basic properties. Formalized Mathematics, 1(1):73-83, 1990.

[26] Xiaopeng Yue, Xiquan Liang, and Zhongpin Sun. Some properties of some special matrices. Formalized Mathematics, 13(4):541-547, 2005.

[27] Katarzyna Zawadzka. The sum and product of finite sequences of elements of a field. Formalized Mathematics, 3(2):205-211, 1992.

[28] Katarzyna Zawadzka. The product and the determinant of matrices with entries in a field. Formalized Mathematics, 4(1):1-8, 1993.

[29] Mariusz Żynel. The Steinitz theorem and the dimension of a vector space. Formalized Mathematics, 5(3):423-428, 1996.

Received May 13, 2008 